PROFESIONALES Y HERRAMIENTAS PARA EL DESARROLLO LOCAL Y SUS SINERGIAS TERRITORIALES. EVALUACIÓN Y PROPUESTAS DE FUTURO IX Coloquio Nacional de Desarrollo Local del GTDL-AGE 

ANTONIO MARTÍNEZ PUCHE, XAVIER AMAT MONTESINOS, ISABEL SANCHO CARBONELL y DANIEL SANCHIZ CASTAÑO (EDS.)

\section{PROFESIONALES Y HERRAMIENTAS PARA EL DESARROLLO LOCAL Y SUS SINERGIAS TERRITORIALES. EVALUACIÓN Y PROPUESTAS DE FUTURO}

IX Coloquio Nacional de Desarrollo Local del GTDL-AGE

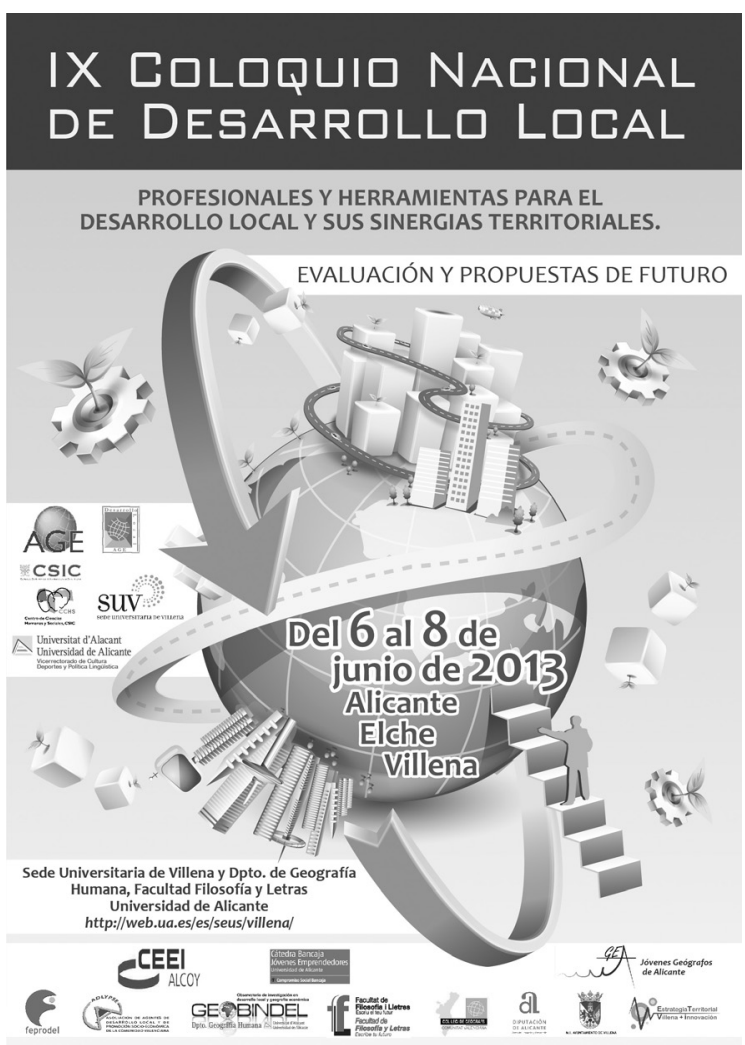


Este libro ha sido examinado y valorado por evaluadores ajenos a la Universidad de Alicante, con el fin de garantizar la calidad científica del mismo.

Publicacions de la Universitat d'Alacant

03690 Sant Vicent del Raspeig

Publicaciones@ua.es

http://publicaciones.ua.es

Telèfon: 965903480

(C) Antonio Martínez Puche, Xavier Amat Montesinos,

Isabel Sancho Carbonell y Daniel Sanchiz Castaño (eds.), 2016

(C) d'aquesta edició: Universitat d'Alacant

ISBN: 978-84-16724-00-0

Dipòsit legal: A 92-2016

Disseny de coberta: candela ink

Composició: Página Maestra (Miguel Ángel Sánchez Hernández)

Impressió i enquadernació: Guada Impresores

\section{unte \\ Unión de Editoriales
Universitarias Españolas \\ WWW.une.es
WWA}

Esta editorial es miembro de la UNE, cosa que garantiza la difusión y comercialización nacional y internacional de sus publicaciones.

Reservados todos los derechos. Cualquier forma de reproducción, distribución, comunicación pública o transformación de esta obra sólo puede ser realizada con la autorización de sus titulares, salvo excepción prevista por la ley. Diríjase a CEDRO (Centro Español de Derechos Repográficos, www.cedro.org) si necesita fotocopias o escanear algún fragmento de esta obra. 


\title{
PROFESIONALES DEL DESARROLLO LOCAL: PASADO, PRESENTE Y, ¿FUTURO INCIERTO O INEXISTENTE? ${ }^{1}$
}

\author{
Ricard Calvo \\ Universitat de València \\ Ricardo.Calvo@uv.es
}

RESUMEN

Si en la década de los ochenta asistimos al nacimiento de una nueva actividad laboral vinculada con la promoción del empleo y el desarrollo local, en las dos décadas posteriores la vimos consolidarse, alcanzando una presencia directa sobre el territorio próxima al noventa por cien del mismo. Pero el actual escenario de recortes supone un cambio sustancial en el proceso de consolidación profesional iniciado, planteando unas perspectivas de futuro poco favorables para este colectivo. En el presente texto proponemos un análisis de los factores que han determinado la situación actual de incertidumbre en la que se encuentra el colectivo de técnicos AEDL. Para ello diferenciaremos entre aquellos factores que conseguidos en este tiempo, con los recortes actuales se han puesto en peligro -incluso se han perdido definitivamente- y aquellos que no se habían conseguido y que por tanto se han convertido en carencias -dificultades y resistencias- de carácter estructural en este proceso.

Palabras clave: AEDL; ADL; desarrollo local.

LOCAL DEVELOPMENT PROFESSIONALS: PAST, PRESENT, FUTURE UNCERTAIN OR ABSENT?

1 Mi agradecimiento a José Manuel González (amigo y compañero AEDL) por la revisión realizada del texto. 


\section{AbSTRACT}

In the eighties we saw the start of a new work activity related to the promotion of employment and local development, in the following two decades we saw the consolidation, reaching a direct presence on the territory close to ninety per cent of it. But the current stage of cuts represents a substantial change in the professional consolidation process started years ago, raising an uncertain future for this group. In this paper, we propose an analysis of the factors behind the current state of uncertainty which is affecting this group of professionals (AEDL - Local Agent of Employment and development). We will study two main factors, one of them are the achieved in this time, and the others were not obtained and therefore have become shortcomings from a structural point of view.

Keywords: AEDL, ADL, local development.

\section{INTRODUCCIÓN: PASADO Y PRESENTE DEL DESARROLLO LOCAL EN ESPAÑA ${ }^{2}$}

Si bien hasta la segunda mitad de la década de los ochenta no encontramos una referencia normativa explícita a los profesionales del desarrollo local, no podemos obviar que sus orígenes están muy ligados, por una parte, a la existencia a partir de 1955 de los Agentes de Extensión Agraria, y por otra, a las Iniciativas Locales de Empleo (I.L.E.) puestas en práctica en España por el INEM a principios de los ochenta (González, 2008; Martínez, 2011).

Es la Orden Ministerial de 21 de febrero de 1986 la que contempla por primera vez entre sus disposiciones una línea de subvenciones destinadas a las entidades locales con la finalidad de financiar hasta el cincuenta por cien de los costes laborales derivados de la contratación por una anualidad, de la contratación de lo que define como agentes de desarrollo, promotores de empleo o expertos de naturaleza análoga que tengan como función la de promover, desarrollar o asesorar iniciativas locales de empleo. Ello permite iniciar la construcción del modelo (ver cuadro 1).

2 El presente texto toma como base empírica de referencia los resultados más destacados obtenidos de una línea de investigación desarrollada desde 2007 en el Departamento de Sociología y Antropología Social de la Universitat de València, en el ámbito de la Comunitat Valenciana, que pretende como objetivo principal conocer los efectos generados sobre el territorio por las actuaciones públicas en la mejora del empleo y el desarrollo local. Dadas las características del proyecto y el tipo de participantes en la investigación, se ha optado a lo largo del mismo por una investigación en la que la recogida de la información se realizara a través de la triangulación de técnicas cuantitativas -cuestionarios y hojas registro de datos diseñados al efecto- con técnicas cualitativas -grupos de discusión y entrevistas a expertos en desarrollo local. 


\section{Cuadro 1. Proceso evolución profesional del técnico AEDL}

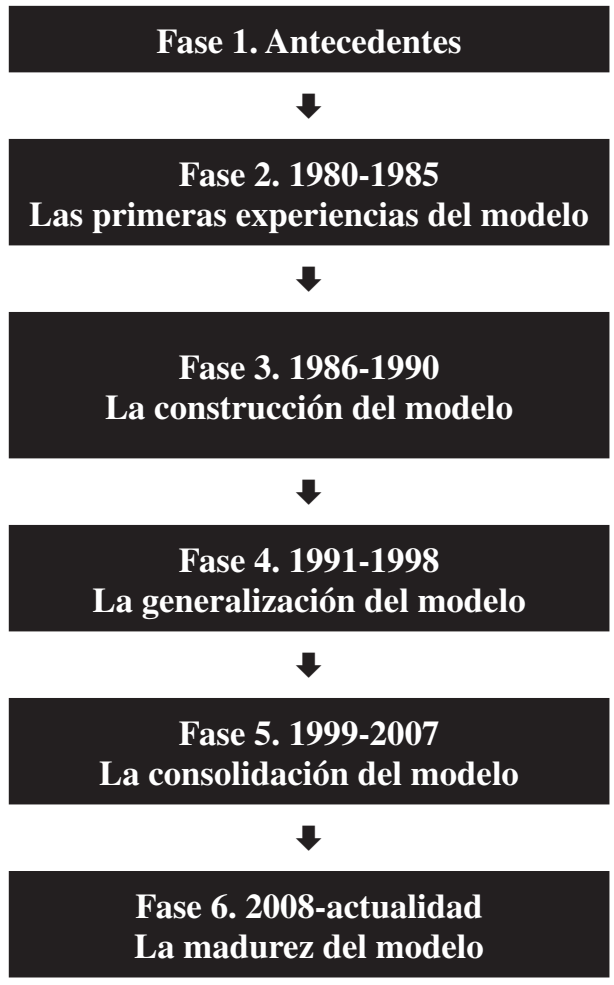

Agentes de Extensión Agraria (1955)

Programas Iniciativas Locales de Empleo (ILE)

Constitución de las primeras

Concejalías de Desarrollo Local y

Promoción Económica

Regulación oficial de la figura del técnico AEDL

Implantación de los AEDL en la mayoría de municipios españoles

Definición de modelos autonómicos propios y creación de redes

Fuente: Calvo y González, 2011.

La aparición de estos técnicos sobre el territorio, gracias en buena medida a la existencia de fondos procedentes de la Unión Europea -a la que España se acababa de incorporar en 1986- permitieron la paulatina generalización del modelo, ya que ello posibilitó a muchos Ayuntamientos plantearse la posibilidad de disponer de un técnico propio que fomentara el empleo y el desarrollo de sus municipios. Esta realidad la vemos de manera cuantitativa en el cuadro 2 con datos referidos a la Comunitat Valenciana.

Este proceso de aparición de los técnicos AEDL coincide con el proceso de descentralización política iniciado de manera intensa a un doble nivel en la década de los noventa: hacia las comunidades autónomas -entendidos como los estamentos gestores de muchas de las políticas públicas- y hacia las entidades locales como las administraciones públicas más próximas a la ciudadanía. Y ello influye decisivamente en la consolidación que el modelo tiene en la década del 2000, donde alcanza la mayor presencia sobre el territorio (ver cuadro 2). 
Cuadro 2. Evolución del modelo de desarrollo en la C. V. (1985-2012)

\begin{tabular}{|c|c|c|c|}
\hline & $\begin{array}{c}\text { Número } \\
\text { AEDL }\end{array}$ & $\begin{array}{c}\mathbf{N}^{\mathbf{0}} \text { de poblaciones con servicio } \\
\text { de AEDL }\end{array}$ & $\begin{array}{c}\text { Tasa de cobertura } \\
\text { de la población }\end{array}$ \\
\hline 1985 & 19 & 12 & $14,42 \%$ \\
\hline 1990 & 48 & 41 & $21,30 \%$ \\
\hline 1995 & 100 & 89 & $33,74 \%$ \\
\hline 2000 & 211 & 165 & $52,90 \%$ \\
\hline 2005 & 495 & 293 & $65,77 \%$ \\
\hline 2010 & 656 & 416 & $89,41 \%$ \\
\hline 2012 & 554 & 352 & $74,78 \%$ \\
\hline
\end{tabular}

Fuente: Calvo, 2011.

La lógica de esta consolidación debería haber dejado paso a una etapa de madurez del modelo en la que este se desarrollara a nivel autonómico de forma plena, pero la situación de crisis y la consiguiente política de recortes han desdibujado este camino, trazando otro en el que las decisiones no va a en la misma dirección.

\section{FutURO: ¿iNCIERTO O INEXISTENTE?}

A tenor de los recortes planteados hasta el momento, con partidas presupuestarias que sufren reducciones de hasta el 65 por cien -como es el caso de la propia que financia la contratación de los técnicos AEDL- e incluso otras que han sido eliminadas casi por completo -muchos programas y actuaciones vinculados con las políticas activas de empleo-, no muestran un futuro demasiado esperanzador ni para el colectivo de técnicos que la desarrolla ni para la actividad como tal.

Y, ¿a qué se debe esta situación?, ¿es debida única y exclusivamente a la reducción de recursos disponibles para el desarrollo local?, o ¿es posible que existan otros factores determinantes en ella?. A partir de las informaciones obtenidas en el estudio de referencia, podemos determinar la existencia de una serie de factores con influencia en este proceso. Entre ellos destacamos la cobertura territorial alcanzada por el modelo como realidad, que ello haya permitido que se convierta en referencia en la materia, y como consecuencia, haya obtenido un reconocimiento universitario que pone de manifiesto su importancia. Pero también factores como la concepción del modelo, que ha influido decisivamen-

3 Tasa de cobertura de la población, que compara la población de los municipios que cuentan con un servicio de AEDL con el total de la población de la Comunitat Valenciana en cada periodo. 
te en muchos de los factores restantes, como el compromiso oficial demostrado con la actividad que no ha supuesto la existencia de un reconocimiento real de la misma, la temporalidad innata con la que ha convivido el modelo, el individualismo (localismo) que ha regido su operatividad a lo largo de estos años, la dependencia de una financiación pública del modelo, la no visión estratégica y de futuro del mismo, y finalmente un conglomerado de factores vinculados con la evolución profesional del colectivo de AEDL.

Antes de presentar con mayor detenimiento cada uno de ellos, apuntamos una característica que los diferencia, ya que los tres primeros factores enunciados son factores que se han conseguido en estos años y que ahora posiblemente se van a perder. Por el contrario, el resto de factores no se han alcanzado en este tiempo y que por tanto tienen una mayor influencia, ya que se convierten en resistencias -carencias estructurales- no conseguidas hasta el momento por el modelo. En la siguiente figura presentamos estos factores de forma gráfica, diferenciándolos en base a su vertiente teórica u operativa.

Cuadro 3. Representación gráfica de los factores determinantes

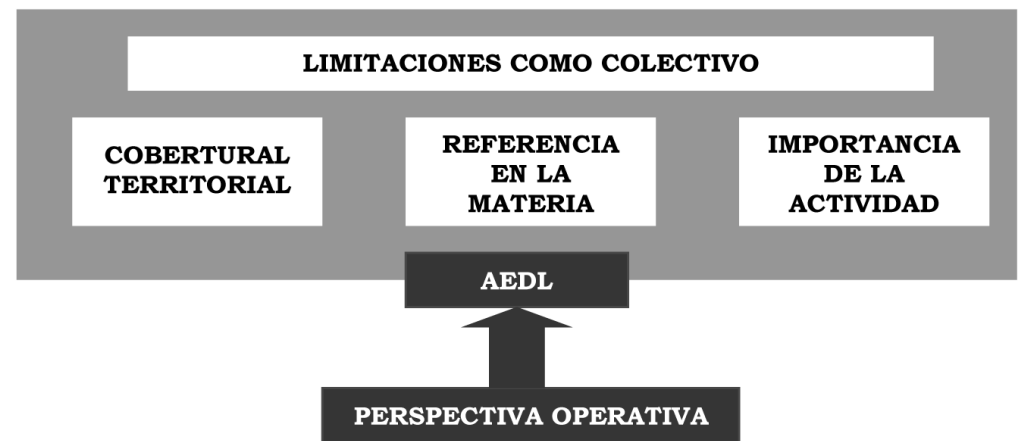

MODELO DE DESARROLLO LOCAL

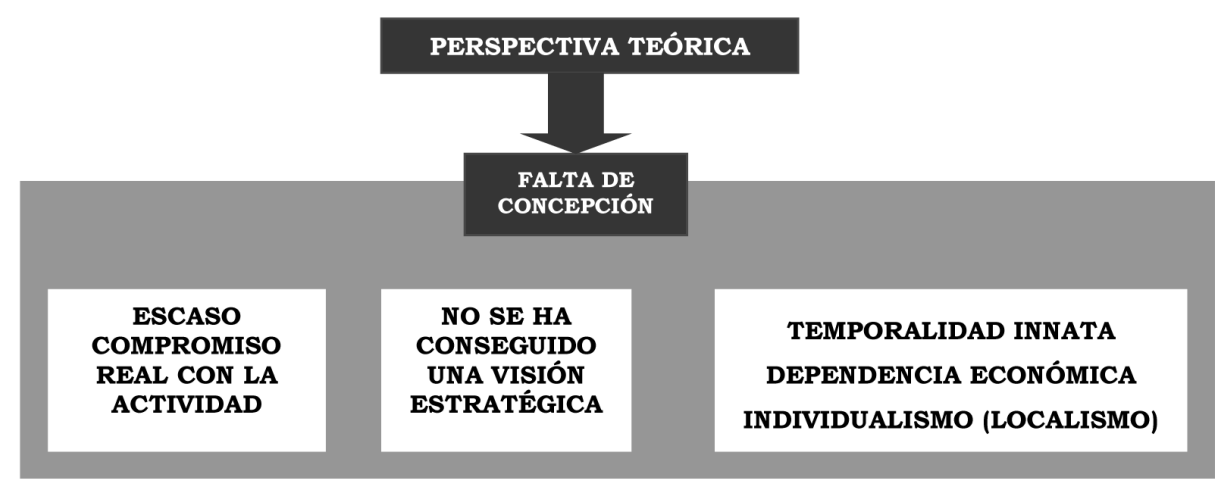

Fuente: Elaboración propia. 
El primero de los factores propuestos hace referencia a la cobertura territorial alcanzada por el modelo, que conforme a los datos presentados en el cuadro 2, pone de manifiesto que el colectivo de AEDL ha alcanzado un número muy representativo sobre el territorio, dando estabilidad y estructura al mismo (Calvo y Martínez, 2012). En este sentido, la realidad del modelo nos muestra la existencia de una comunidad representativa tanto en su vertiente cuantitativa, manifestada principalmente en el número de técnicos existentes (recordemos cifrada en 2010 en la C.V. en una cantidad próxima a los 656 técnicos), como en su vertiente cualitativa, analizada a través de su presencia sobre el territorio (los citados técnicos desarrollan su actividad en 416 municipios de los 542 del ámbito analizado - un 76,8 por cien del total de los mismos- lo que ha supuesto en términos de cobertura directa que el modelo implantado haya alcanzado un 89,41 por cien de la población, llegándose a la totalidad del mismo si nos referimos a la cobertura indirecta). Por tanto han tenido presencia, y además una presencia que se ha caracterizado por la proximidad del servicio, lo que se ha convertido en un rasgo definitorio del modelo, jugando un papel fundamental la accesibilidad del usuario al servicio. Accesibilidad del usuario al servicio que ahora se pone en entredicho con grandes posibilidades de que este se reduzca drásticamente o incluso se elimine por completo.

Este crecimiento continuo ha posibilitado que se hayan convertido en un referente en la materia. El tiempo, el trabajo desarrollado y la experiencia adquirida han convertido al colectivo de AEDL, en su condición de máximo exponenete del modelo de desarrollo desde lo local, en un colectivo con una voz autorizada en la materia, que saben de lo que hablan (tal y como se autocatalogan). La experiencia se convierte en un grado que les permite calificarse como expertos en desarrollo local, aunque esta experiencia haya sido adquirida a fuerza de hacer y deshacer, y de una dinámica de trabajo basada en la pruebaerror, donde no han existido unas directrices claras previas, que ayudaran a desarrollar coherentemente su actividad. A ello cabe añadir que no han tenido competencia en su materia sobre el territorio, ya que no existe ningún otro técnico dedicado al empleo y al desarrollo local. Ello ha posibilitado, como hemos dicho, un crecimiento tanto en la cantidad como en los contenidos de su trabajo, pasando de ser meros ejecutores de programas de empleo a implementar programas de desarrollo local. La figura del técnico AEDL es una figura concebida desde sus orígenes funcionalmente para la implantación de actuaciones a nivel local, es decir, en contacto directo con la esencia de cualquier proceso de desarrollo, el binomio territorio-población. Se trata este de un objetivo que se ha cumplido a la perfección, ya que con el paso de los años la importancia de lo local -y por tanto del modelo y de los técnicos- en la gestión y desarrollo de las políticas activas para el empleo se ha ido acrecentando hasta el punto de poder afirmar que en el período comprendido entre 2008-2011, casi el 60 por cien de las actuaciones emprendidas en la Comunitat Valenciana vinculadas 
con el empleo han sido lideradas por el nivel local, y por tanto por las Agencias locales donde operaban estos técnicos AEDL (Calvo, 2011).

La importancia de la labor desarrollada en este tiempo se manifiesta entre otros gracias al reconocimiento generado desde los estamentos universitarios, a través de una formación de postgrado. No podemos olvidar que ha sido tradicionalmente la existencia de unos estudios académicos de referencia la acreditación válida más visible que hemos podido encontrar para la configuración profesional de cualquier actividad. En el caso que nos ocupa ha sido este un requisito de difícil cobertura al tratarse de una actividad con un carácter multidisciplinar, que requiere de visiones aportadas desde distintas y variadas perspectivas, tales como la Economía, la Geografía, el Derecho o la Psicología entre otras. Este aval universitario no hace más que hacer explícito el reconocimiento del conocimiento adquirido y puesto en práctica por estos técnicos, su how-know específico.

¿Qué es desarrollo local?, o ¿qué no es desarrollo local? Es una de las cuestiones no resueltas hasta el momento. La inexistencia de una concepción clara del desarrollo como paradigma y del modelo como aplicación, han derivado en una realidad que nos muestra una indefinición genérica, que no ayuda a determinar los objetivos perseguidos por el mismo. ¿Cuáles son? o ¿dónde se especifican?, son preguntas sin respuesta transcurridos más de veinticinco años. Tanto ha sido así que las funciones asignadas a los técnicos AEDL enunciadas en el articulado de la Orden de 15 de julio de $1999^{4}$, son lo más parecido a unos objetivos que podemos encontrar para el mismo, por lo que, la pregunta que nos hacemos es: ¿hemos de entender que los objetivos del modelo son las funciones que se les asignan a los técnicos AEDL?. La no existencia de estos objetivos, supone en sí misma una carencia estructural del proceso de gestión

4 Orden por la que se establecen las bases de concesión de subvenciones públicas para el fomento del desarrollo local e impulso de los proyectos y empresas calificados como I+E (modificada parcialmente por la Orden de 27 de diciembre de 1999, por la Orden 49/2005, de 14 de enero y por la Orden TAS/360/2008, de 6 de febrero). Conforme a lo establecido en su artículo 8 se establecen como funciones a desarrollar las siguientes: 1. Prospección de recursos ociosos o infrautilizados, de proyectos empresariales de promoción económica local e iniciativas innovadoras para la generación de empleo en el ámbito local, identificando nuevas actividades económicas y posibles emprendedores; 2. Difusión y estímulo de potenciales oportunidades de creación de actividad entre los desempleados, promotores y emprendedores, así como instituciones colaboradoras; 3. Acompañamiento técnico en la iniciación de proyectos empresariales para su consolidación en empresas, los proyectos generadores de nuevos empleos, asesorando e informando sobre la viabilidad técnica, económica y financiera $\mathrm{y}$, en general, sobre los planes de lanzamiento de las empresas; 4. Apoyo a promotores de las empresas, una vez constituidas éstas, acompañando técnicamente a los mismos durante las primeras etapas de funcionamiento, mediante la aplicación de técnicas de consultoría en gestión empresarial y asistencia en los procesos formativos adecuados para coadyuvar a la buena marcha de las empresas creadas; 5. Y cualesquiera otras que contribuyan a la promoción e implantación de políticas activas de empleo e impulse la creación de actividad empresarial. 
de la actividad desarrollada por el técnico, lo que ha permitido la aparición de interpretaciones particulares e interesadas por parte de cada uno de los agentes actuantes implicados en el modelo o de sus gobernantes en cada momento.

Pese a que el modelo se ha consolidado, creciendo ininterrumpidamente hasta 2010-2011, ello no se ha traducido en un compromiso real, ni en una apuesta clara desde los estamentos gubernamentales por el desarrollo ni por el modelo. Buena muestra de ello es si bien en todo este tiempo la administración local ha asumido competencias en empleo y desarrollo local, lo ha hecho de manera sobrevenida, sin que haya existido ningún proceso de delegación expreso de las mismas. Ello conlleva un doble riesgo a efecto práctico: en primer término, como ya ha sucedido en algunos casos, que un Ayuntamiento pueda desmantelar el servicio desarrollado durante años por la AEDL de una semana para otra acogiéndose a esta no tenencia de las competencias formales al efecto, y en segundo, tal y como parece que finalmente ocurrirá estas competencias van a ser atribuidas formalmente a un nivel supralocal, posiblemente provincial.

Una buena muestra de esa falta de compromiso real con la actividad ha sido su rasgo de temporalidad innata. La dinámica desarrollada en este tiempo por el modelo basada en el parcheado continuo de programas de actuación, ha dibujado un escenario de fuerte inseguridad, como se ha puesto de manifiesto en el momento actual. Un escenario de actuación cortoplacista que ha dificultado en gran medida la articulación de un plan de desarrollo del territorio a medio y a largo plazo.

Junto a ello, la aparición de localismos en el desarrollo del modelo es otro de los factores a tener muy en cuenta. Por un lado, hemos asistido a la aparición de un localismo mal entendido, que ha originado que los límites geográficos de la localidad hayan supuesto en muchos casos un elemento potenciador de una visión totalmente endógena, provocando un individualismo máximo en las actuaciones emprendidas. Ello ha dificultado en gran medida el desarrollo integral del territorio, en este caso entendido, como un territorio más amplio al de la localidad -agrupación de varios municipios con los que se pudiera tener afinidades económicas, sociales, productivas, territoriales, etc., mancomunidad, comarca, provincia o la misma comunidad autónoma incluso. Se confunde por tanto, local (territorio) con localidad (límites municipales). La realidad nos muestra una competencia entre territorios cercanos encubierta, donde los intereses -visibles u ocultos- al desarrollo de determinados programas, o la simple competencia entre entidades vecinas pueden estar en la base del mismo. Ello ha derivado incluso en rivalidades sobre todo entre municipios cercanos para la implementación de actuaciones. Tampoco ha existido un proceso de aprendizaje colectivo, ni una sinergia profesional, faltando una plataforma donde compartir la experiencia -y por tanto las experiencias generadas. En general, en el caso de las Agencias locales y los técnicos AEDL operantes, el proceso de generación de técnicas específicas para el desarrollo de su actividad no ha 
sido uno de sus rasgos característicos. Si bien estas técnicas existen desde un punto de vista aplicado, no se han constituido como herramientas de trabajo para el colectivo. Ha tenido un proceso concreto, ya que se han generado mayoritariamente a nivel micro, local e individual, en un plano de informalidad muy significativo.

En este sentido la financiación del modelo es otro elemento a considerar en este repaso, ya que la dependencia extrema generada por el mismo de las subvenciones y ayudas públicas (procedentes de fondos europeos, nacionales, autonómicos o en el mejor de los casos locales) ha limitado la capacidad de hacer del modelo hasta el punto que en un momento como el actual, en el que las dotaciones públicas se han reducido drásticamente, la actividad ha quedado huérfana momentáneamente. Este clientelismo subvencionado ha sido máximo en la figura de los propios técnicos, siendo su origen subvencionado el mejor de los indicadores de cómo desde un principio ha existido una dependencia económica directa de las subvenciones por parte del modelo (Calvo, 2011). Ello se ha convertido en un rasgo muy característico del modelo, aspecto que ha perdurado en el tiempo hasta nuestros días.

También destacamos la dependencia del poder político actuante, tanto a nivel local como supralocal. Factor que ha limitado en gran medida la puesta en práctica de una visión estratégica del desarrollo. Una doble sujeción-dependencia al poder político se ha articulado en el modelo: la falta de concreción de la actividad por un lado, y por otro, la ausencia de autonomía en el desarrollo del trabajo. Ello responde a una dependencia en origen potenciada por la falta de determinación de las competencias específicas de la actividad y/o del técnico en la esfera local, que lo ha desprovisto de la capacidad, el estatus y el reconocimiento suficiente dentro de la Administración municipal. Ello ha provocado una dependencia sobrevenida por el paso del tiempo que no ha hecho sino agravar la situación (premisa anterior). Por lo que el modelo ha quedado en muchos casos a merced del político asignado -Concejal, responsable, director o el propio Alcalde en municipios pequeños- que han ido determinando, y en muchos casos acotando su capacidad de hacer. Ello ha generado con el paso de los años una desvaloración de la actividad en general y concretamente de la labor desarrollada por el técnico. Además es en sí misma una dependencia procedente de las trabas del propio funcionamiento de la administración pública, que han desprovisto al técnico de una autonomía operativa, gestional y decisoria suficiente en el desarrollo de su actividad.

Finalmente también cabe mencionar como factor a tener en cuenta que aparecen una serie de limitaciones vinculadas con la actividad procedentes del propio colectivo profesional. Estos años de actuación, han caracterizado al colectivo de técnicos AEDL por un lado, por no haber conseguido una autopercepción colectiva común, y por otro, por no haber sabido dar a conocer su actividad (venderla socialmente como indicaba una de las técnicas entrevis- 
tadas). La no existencia de un consenso interno entre los técnicos, ha tenido unos efectos perversos sobre su proceso de profesionalización, bien impidiendo la determinación de un imaginario de competencias propio e interno de este colectivo, bien no permitiendo el desarrollo técnico natural de la actividad y ralentizando el proceso de generación de técnicas y herramientas específicas que ayuden a consolidar la profesión en el mercado laboral. También ha dificultado la valoración que la sociedad en general ha realizado de su utilidad, dado que el conocimiento social de la labor desarrollada por estos técnicos ha sido mínimo, pasando inadvertida en gran número de los casos. En ello ha tenido mucha importancia la no existencia de un adecuado proceso de retroalimentación de los resultados obtenidos por ellos hacia su contexto básico de actuación concretado en los usuarios, administraciones implicadas y sociedad en general. Y el escaso grado de publicidad que se da habitualmente a los resultados obtenidos por las agencias en la aplicación de los programas de empleo y desarrollo local, tanto a nivel interno-institucional como a nivel externo o social. Debido a razones de naturaleza muy diversa, tales como: a) inexistencia de datos, ya que no se mide, al menos formalmente; b) falta de costumbre; c) el político se apropia de los méritos positivos de los programas; d) el tipo de resultados a ofertar, ¿todos? ¿los políticamente correctos?; e) el grado de respaldo por parte del político de la labor desarrollada por el técnico; f) el posicionamiento de la agencia dentro del municipio, ¿está reconocida su labor?; y g) ¿tiene utilidad?.

\section{A MODO DE CONCLUSIÓN FINAL}

A tenor de lo expuesto, tres serían las anotaciones finales que propondríamos a modo de conclusión de este texto. En primer término, la necesidad de aprovechar el momento actual para adecuar el modelo y que este sea válido, ya que el momento deviene en decisivo (ahora o nunca, como nos apuntaba otro técnico). En segundo, e intrínsecamente ligado a lo anterior, hay que articular políticas adaptadas, transversales y útiles para el desarrollo del territorio abandonando la perspectiva anterior de actuaciones genéricas que tan sólo pretendían incidir sobre el empleo. Y por último la necesidad de que las actuaciones aporten una concepción del binomio territorio-población como un todo -visión integral-, que requiera de todos y cada uno de los recursos presentes en él -visión integrada- y que busque entre sus finalidades la obtención de efectos sinérgicos -visión integradora- son los principales fundamentos de cualquier política pública de intervención.

BIBLIOGRAFÍA

CALVO PALOMARES, R. (2011): AEDL (Agente de Empleo y Desarrollo Local): una aproximación sociológica al estudio de una profesión. PUVPublicacions de la Universitat de València. Valencia. 
CALVO PALOMARES, R. y GONZÁLEZ CARDONA, J. (2011): "La creación de empresas de economía social en el modelo de desarrollo local: reflexiones sobre la divergencia actual del caso valenciano". Revista REVESCO: Revista de estudios cooperativos, núm. 104, pp. 7-37.

CALVO PALOMARES, R. (2012): AEDL (Agente de Empleo y Desarrollo Local): Un estudio de su realidad profesional, Alzira, Editorial Germanía.

CALVO PALOMARES, R. y MARTINEZ PUCHE, A. (2012): "Empleo y desarrollo desde lo local: un análisis DAFO de 25 años de actividad de los AEDL en la Comunitat Valenciana”, en Martínez-Puche, A. y Calvo, R. (coords.) (2012): Valoraciones técnicas y repercusiones territoriales sobre el ejercicio profesional del desarrollo local”. Editorial Germania, Alzira (Valencia), pp. 147-201.

GONZALEZ CARDONA, J. (2008): «El agente de empleo y desarrollo local: competencias profesionales y trabajo en red», en Fuertes, A.M. y García, L., De la economía global al desarrollo local. Valencia: Publicacions de la Universitat de València.

MARTÍNEZ PUCHE, A. (2011): El desarrollo local en un contexto globalizado. Cohesión territorial, gobernanza, políticas públicas e instrumentos de innovación. Estudios de casos en el Corredor Industrial del Vinalopó (Alicante), Colección Tesis Doctorales, taller digital de la Universidad de Alicante, Alicante. 